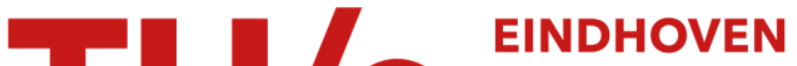 \\ UNIVERSITY OF \\ TECHNOLOGY
}

\section{Increasing beamsteering directions using polyphase decomposition}

Citation for published version (APA):

Schobben, D. W. E., \& Sommen, P. C. W. (1996). Increasing beamsteering directions using polyphase decomposition. In Proc. IEEE Signal Processing Workshop on Statistical Signal and Array Processing (pp. 117120). IEEE Signal Processing Society.

Document status and date:

Published: 01/01/1996

\section{Document Version:}

Accepted manuscript including changes made at the peer-review stage

\section{Please check the document version of this publication:}

- A submitted manuscript is the version of the article upon submission and before peer-review. There can be important differences between the submitted version and the official published version of record. People interested in the research are advised to contact the author for the final version of the publication, or visit the $\mathrm{DOI}$ to the publisher's website.

- The final author version and the galley proof are versions of the publication after peer review.

- The final published version features the final layout of the paper including the volume, issue and page numbers.

Link to publication

\section{General rights}

Copyright and moral rights for the publications made accessible in the public portal are retained by the authors and/or other copyright owners and it is a condition of accessing publications that users recognise and abide by the legal requirements associated with these rights.

- Users may download and print one copy of any publication from the public portal for the purpose of private study or research.

- You may not further distribute the material or use it for any profit-making activity or commercial gain

- You may freely distribute the URL identifying the publication in the public portal.

If the publication is distributed under the terms of Article $25 \mathrm{fa}$ of the Dutch Copyright Act, indicated by the "Taverne" license above, please follow below link for the End User Agreement:

www.tue.nl/taverne

Take down policy

If you believe that this document breaches copyright please contact us at:

openaccess@tue.nl

providing details and we will investigate your claim. 


\title{
Increasing Beamsteering Directions Using Polyphase Decomposition
}

\author{
Daniël W.E. Schobben and Piet C.W. Sommen \\ Department of Electrical Engineering, Eindhoven University of Technology \\ P.O. Box 513, 5600 MB Eindhoven, The Netherlands \\ D.W.E.Schobben@ele.tue.nl
}

\begin{abstract}
In many implementations of digital delay and sum beamforming, a sample rate much higher than the Nyquist rate is used. This allows for many synchronous beamsteering directions. Severe demands are made upon the analogue to digital converters however. Several methods have been proposed for reducing the sample rate required. These methods incorporate the delays that are needed for beamforming in time domain [3], [4] or in frequency domain [5]. A more efficient method for implementing a time-domain delay and sum beamformer using polyphase decomposition is presented in this paper. This method results in significant computational savings when the desired angular resolution is high compared to the number of sensors used and the number of simultaneously formed beams.
\end{abstract}

\section{Introduction}

Conventional continuous-time beamformers delay all sensor outputs so that propagation delays are cancelled and the sensor outputs can be combined coherently. In a discretetime beamformer, these delays are performed digitally. Using discrete time delays only allows for delaying over an integer multiple of the sampling time period. Therefore, the number of synchronous beam-pointing directions is small for low sample rates, resulting in a poor angular resolution. To illustrate this, it is shown in Section 2 that a linear array sampled at $v$ times the Nyquist rate can only be steered to $1+2 v$ synchronous angles. A signal arriving from a non-synchronous direction can be received by steering the beam to the most nearby synchronous angle or by rounding the delays needed for beamforming to the delays available. Both methods introduce severe distortion and poor spatial discrimination for small $v$. In Section 3 the concept of interpolation beamforming is discussed. This technique uses interpolation, so that the sampling rate is increased artificially. In this way, delays are obtained which are a fraction of the unit delay [2]. In Section 4 an efficient method is presented for the implementation of the interpolation beamformer using polyphase decomposition. The resulting complexity is discussed in Section 5 and a numerical example shown in Section 6.

\section{Linear Sensor Array Beamforming}

Although the method to be presented can be applied to all array geometries, an example of the steering capabilities of a linear array is discussed. For a linear array the anticipated propagation delay of a flat wave plane from the first sensor to the $i^{t h}$ sensor equals

$$
\tau_{\max }-\tau_{i}=\frac{i d \sin \theta}{c},
$$

where $d$ is the sensor inter-distance, $\theta$ is the direction of arrival (DOA) relative to broadside (the direction perpendicular to the line-array), $c$ is the wave plane propagation velocity and $\tau_{\max }$ is the propagation delay of the wave plane between the first and the last sensor. The wave plane incident to the linear array is depicted in Figure 1. The sensor outputs

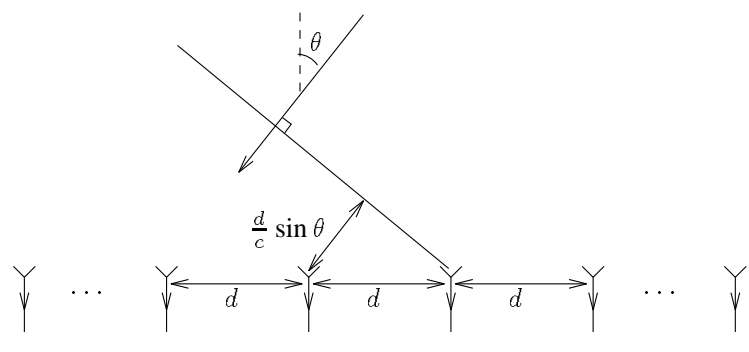

Figure 1. Wave front incident to a linear array

are sampled at the rate $f_{s}=\frac{1}{T_{\mathrm{s}}}$ and consequently the beam can only be steered to the angles which yield a difference in propagation delay of $u T_{s}$ seconds between neighboring sensors, with $u$ an integer. This delay is cancelled using 
time-delays $u T_{s}=\frac{d \sin \theta}{c}$. The beam can thus be steered to

$$
\theta_{u}=\sin ^{-1}\left(\frac{c u T_{s}}{d}\right)
$$

A common choice for $d$ that prevents spatial aliasing is $d=\frac{\lambda_{0}}{2}, \lambda_{0}$ being the minimum wavelength of the signal to be received. For this sensor inter-distance and a sample rate equaling $f_{s}=2 v f_{0}$, it follows from (2) that $|u| \leq v$. There exist $1+2\lfloor v\rfloor$ different $u$ that obey this equation, so the beam can be steered to $1+2\lfloor v\rfloor$ different angles. For example, when the sample rate for equals $4 f_{0}(v=2)$, it follows from (2) that the beam can only be steered to $0, \pm 30^{\circ}$ and $\pm 90^{\circ}$. A higher angular resolution can be achieved by interpolating the sampled data. When $v=2$ and more than 5 synchronous directions are desired, the data can be interpolated by a factor $M$. This is depicted in Figure 2 for $M=4$. The solid lines indicate the sampled

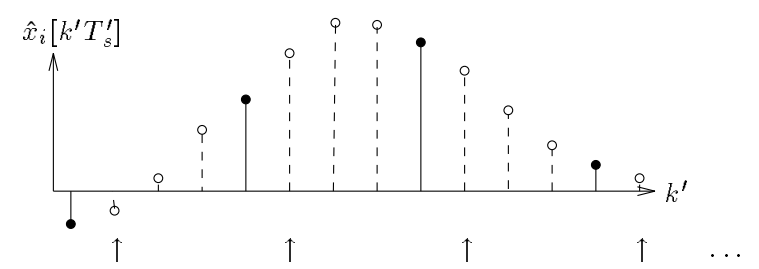

Figure 2. Data interpolation

data and the dashed lines indicate the interpolated data. A delay of $\frac{3 T_{s}}{4}$ for example can now be achieved by selecting the interpolated samples indicated with an $\uparrow$ in Figure 2. Interpolating with a factor $M=4$ now allows for beamsteering to $0, \pm 7.18^{\circ}, \pm 14.5^{\circ}, \pm 22.0^{\circ}, \pm 30.0^{\circ}, \pm 38.7^{\circ}$, $\pm 48.6^{\circ}, \pm 60.0^{\circ}, \pm 90.0^{\circ}$. Clearly, only one of every $M$ interpolated samples is used for beamforming.

\section{Interpolation Beamforming}

The interpolation process for a single beam is depicted in Figure 3. First the sensor data is sampled at a rate equal to or exceeding the Nyquist sampling rate. The $i^{\text {th }}$ sampled sensor output $x_{i}\left[k T_{s}\right]$ is zero padded to obtain $\tilde{x}_{i}\left[k^{\prime} T_{s}^{\prime}\right]$

$\tilde{x}_{i}\left[k^{\prime} T_{s}^{\prime}\right]=\left\{\begin{array}{ll}x_{i}\left[\frac{k^{\prime}}{M} T_{s}\right], & \text { for } k^{\prime}=0, \pm M, \pm 2 M, \ldots \\ 0, & \text { otherwise }\end{array}\right.$,

with $T_{s}^{\prime}=\frac{T_{s}}{M}$. Then the $\tilde{x}_{i}$ are filtered with the interpolation filter $H$ to obtain the $\hat{x}_{i}$

$$
\hat{x}_{i}\left[k^{\prime} T_{s}^{\prime}\right]=\sum_{l=0}^{L-1} h\left[l T_{s}^{\prime}\right] \tilde{x}_{i}\left[\left(k^{\prime}-l\right) T_{s}^{\prime}\right] .
$$

This filter is a Finite Impulse Response filter with impulse response $h\left[k^{\prime} T_{s}^{\prime}\right]$. The $\hat{x}_{i}$ are delayed over $p_{i} T_{s}^{\prime}$ seconds, to compensate for the anticipated propagation delays, where the $p_{i} T_{s}^{\prime}$ are equal to the $\tau_{i}$. Then down-sampling is used to obtain

$$
x_{i}^{\prime}\left[k T_{s}\right]=\hat{x}_{i}\left[\left(k M-p_{i}\right) T_{s}^{\prime}\right] .
$$

The beamformer output $y\left[k T_{s}\right]$ is obtained by summing the shaded $x_{i}^{\prime}$. Shading means multiplying the $x_{i}^{\prime}$ with weights to enhance the angular discrimination. To simplify notation, the shading is not mentioned explicitly in figures and equations. Multiple beams can be formed from the interpolated sensor outputs $\hat{x}_{i}$ without performing additional multiplications.

Pridham and Mucci [3] argued that the scheme of Figure 3 is equal to the scheme in Figure 4 for the case that only one beam is formed. This can be seen by interchanging the filter $H$ and the delays $p_{i} T_{s}^{\prime}$ and placing the filter $H$ and the down-sampling in Figure 3 after the summation. This is allowed when all filters are identical, linear and time invariant. Furthermore, the filter and the down-sampling may be combined to reduce complexity. It will be shown in Section 5 that the complexity of the technique proposed in the following section is lower than that of the scheme in Figure 4 for high angular precision beamformers. Note that forming multiple beams is not possible without performing additional multiplications with this scheme.

\section{Polyphase Decomposition}

In this section an efficient implementation of the interpolation beamformer is presented. First, consider the data processing in Figure 3 for $i^{t h}$ sensor only. This is depicted in Figure 5(a). Here the delay $p_{i} T_{s}^{\prime}$ is interchanged with interpolation filter $H$. This is allowed since $H$ is linear and time invariant. In Figure 5(b) filter $H$ is decomposed into the filters $H_{0}, H_{1} \ldots H_{M-1}$ using polyphase decomposition [1]. The impulse responses of the $H_{j}$ can be calculated from the impulse response of $H$ according to

$$
h_{j}\left[k^{\prime} T_{s}^{\prime}\right]=\left\{\begin{array}{ll}
h\left[\left(k^{\prime}+j\right) T_{s}^{\prime}\right], & \text { for } k^{\prime} \in \kappa \\
0, & \text { otherwise }
\end{array},\right.
$$

for $j=0,1, \ldots, M-1$ and $\kappa=\left\{0, M, \ldots, M\left\lfloor\frac{L-j-1}{M}\right\rfloor\right\}$. Down-sampling these filter outputs is equivalent to downsampling the data and then filtering with $H_{j}^{\prime}$, as depicted in Figure 5(c). The impulse responses of the $H_{j}^{\prime}$ are given by

$$
h_{j}^{\prime}\left[k T_{s}\right]=h_{j}\left[k M T_{s}^{\prime}\right],
$$

for $k=0,1, \ldots,\left\lfloor\frac{L-j-1}{M}\right\rfloor$. Up-sampling with a factor $M$, delaying over $p_{i} T_{s}^{\prime}$ and down-sampling with a factor $M$ is equal to delaying over $\frac{p_{i}}{M} T_{s}$ if $p_{i}$ is an integer multiple of 
$M$, and equal to zero otherwise. Therefore Figure 5(c) can be interpreted as choosing the filter $H_{q}^{\prime}$ and delaying over $r T_{s}$, where

$$
\begin{aligned}
q & =M\left\lceil\frac{p_{i}}{M}\right\rceil-p_{i}, \\
r & =\frac{q+p_{i}}{M}=\left\lceil\frac{p_{i}}{M}\right\rceil .
\end{aligned}
$$

The resulting scheme is depicted Figure 5(d). The combination of the delay of $r T_{s}$ and the sub-filter $H_{q}^{\prime}$ represents an approximation of the desired delay $p_{i} T_{s}^{\prime}$. The sub-filter must be of sufficient length to guarantee a good approximation of the desired delay. In contradiction to this demand, a long sub-filter requires many multiplications per second, and introduces a long beamformer delay.

\section{Comparison of Computational Complexity}

As a measure of complexity, the number of multiplications per seconds of the interpolating filter is considered. The filter $H$ is assumed to be of length $L=w M$ throughout this section, with $w$ integer. Although this is not necessary, it gives more insight in the calculation of the complexity. The proposed beamformer is compared with the beamformers of Figure 3 and 4.

In the scheme of Figure 3, $N$ filters of length $L$ are calculated at a rate $f_{s}^{\prime}$, with $N$ the number of sensors. Using $f_{s}^{\prime}=M f_{s}$ and taking advantage of the sparse input data of the interpolation filters, this yields a complexity of $L N f_{s}$ multiplications per second. This complexity is independent of the number of beams to be calculated, and is therefore efficient when a large number of beams is required. Furthermore, assuming that the filter $H$ is a linear phase filter, the number of multiplications per second can be reduced by approximately a factor 2 , yielding a complexity of $\frac{L N f_{s}}{2}$ multiplications per second.

Filter $H$ in Figure 4 is calculated for each beam at a rate $f_{s}$, since only one of every $M$ samples is needed. The number of multiplications per second equals $L N_{B} f_{s}$, with $N_{B}$ the number of beams to be calculated. Again, assuming that the interpolation filter is a linear phase filter, the resulting number of multiplications per second equals $\frac{L N_{B} f_{s}}{2}$.

The scheme in Figure 4 is more efficient than the scheme in Figure 3 if and only if the number of beams to be calculated is smaller than the number of sensors $\left(N_{B}<N\right)$.

In the proposed beamformer, sub-filters $H_{q}^{\prime}$ are of length $w$. For each beam, $N$ sub-filters are calculated at a rate $f_{s}$. The total number of multiplications per second equals $w N N_{B} f_{s}=\frac{L N N_{B} f_{s}}{M}$. In general, it is not possible to exploit the linear phase property of the interpolation filter to reduce the complexity further. When $M<2 N_{B}$ and $N<N_{B}$ interpolating all sensor data as in Figure 3 is most efficient. Combining all interpolation filters as in Figure 4 is most efficient when $M<2 N$ and $N>N_{B}$. Thus the proposed method outperforms its alternatives when a high angular resolution is required $\left(M>2 N\right.$ and $\left.M>2 N_{B}\right)$. This scheme has a gain in computational complexity over its alternatives of $\frac{M}{2 N}$ and $\frac{M}{2 N_{B}}$ respectively. The quality of the delays formed depends on the sub-filter length $w=\frac{L}{M}$. When both the filter length $L$ and the interpolation factor $M$ are increased with the same factor, the number of beam-pointing directions further increases while the computational complexity does not increase for the proposed method. Consequently, the angles for which beams can be formed can now be chosen with arbitrarily precision while maintaining the same amount of multiplications per second. In practice, the filter length $L$ is limited however since $\frac{L}{2}$ tab weights are stored into a finite amount of memory. For the two alternative methods the computational complexity does however increase proportionally with the filter length $L$.

\section{Example of Computational Complexity}

Next, an example is given to show that the conditions for the proposed method to be more efficient than its alternatives are easily met. Consider sub-filters of length $w=10$, $N=7$ sensors, $M=20$ (41 different synchronous angles) and $N_{B}=5$ (five beams are formed). The main lobes of the unshaded beam-patterns corresponding to the resulting synchronous beam-pointing directions are depicted in Figure 6 for $\theta$ in between $0^{\circ}$ and $90^{\circ}$. For negative angles, the figure is symmetrical. The figure shows that there indeed is a need for a high $M$ to exploit the best possible angular discrimination. However, when $M$ is chosen much larger, the angular discrimination no longer improves, as the successive beams merely overlap. In practice, $M$ will be in between 15 and 40 for a 7 sensor array which is sampled at the Nyquist rate, and the proposed method outperforms the alternatives discussed. The complexity for the proposed beamformer equals $w N N_{B} f_{s}=350 f_{s}$ multiplications per second for this example. The alternate schemes of Figure 3 and 4 require $\frac{L N f_{s}}{2}=700$ and $\frac{L N_{B} f_{s}}{2}=500 f_{s}$ multiplications per second respectively. A significant efficiency gain is thus obtained.

\section{Conclusions and Future Research}

A new method using polyphase decomposition was proposed for reduced complexity interpolation delay and sum beamforming. Significant computational savings are reported for beamformers with a high angular discrimination.

In future research the polyphase equivalent scheme will be used to study relations between interpolation beamforming and other broadband array processing techniques. The use of an adaptive algorithms to track moving sources us- 
ing delay and sum beamformers will also be considered in future research.

\section{References}

[1] A.N. Akansu and R.A. Haddad. Multiresolution Signal Decomposition. London: Academic Press, 1992.

[2] T.I. Laakson et al. Splitting the unit delay. IEEE Signal Proc. Mag., 13(1):30-60, Jan. 1996.

[3] R.G. Pridham and R.A. Mucci. A novel approach to digital beamforming. J. Acoust. Soc. Am., 63:425-434, Feb. 1978.

[4] R.W. Schafer and L.R Rabiner. A digital signal processing approach to interpolation. Proc. IEEE, 61:692-720, Jun. 1973.

[5] M.E. Weber and R. Heisler. A frequency-domain algorithm for wideband coherent signal processing. J. Acoust. Soc. Am., 76:1132-1144, Oct. 1984.

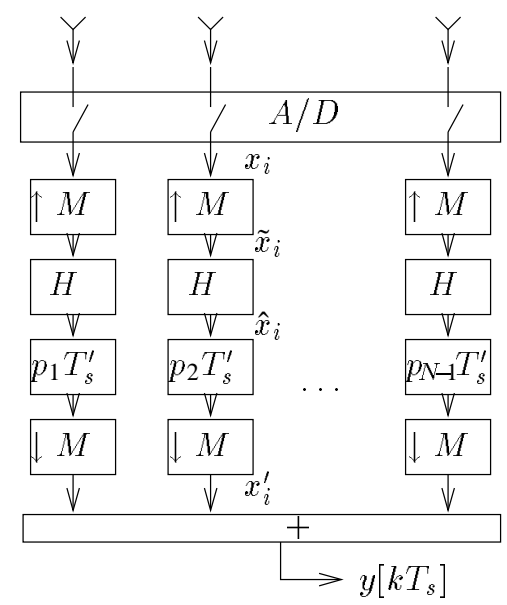

Figure 3. Interpolation beamformer

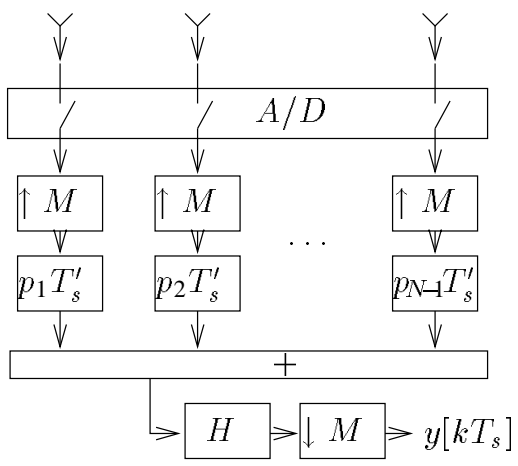

Figure 4. Beamformer equivalent

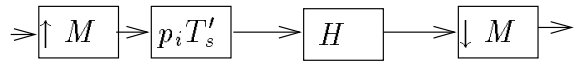

(a)

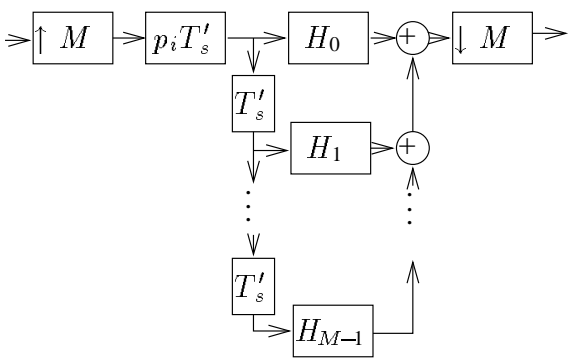

(b)

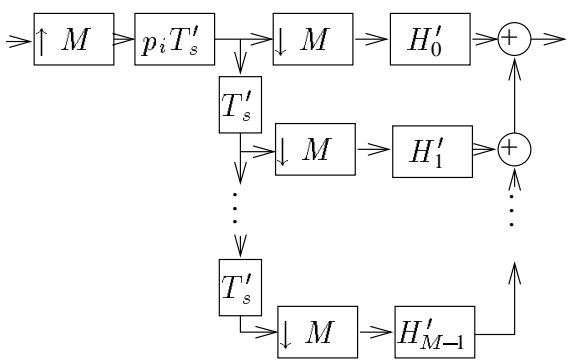

(c)

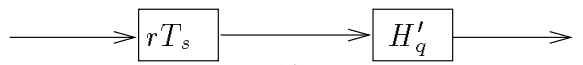

(d)

Figure 5. Interpolation filter (a), polyphase decomposed filter (b), polyphase equivalent (c) and compacted polyphase equivalent (d).

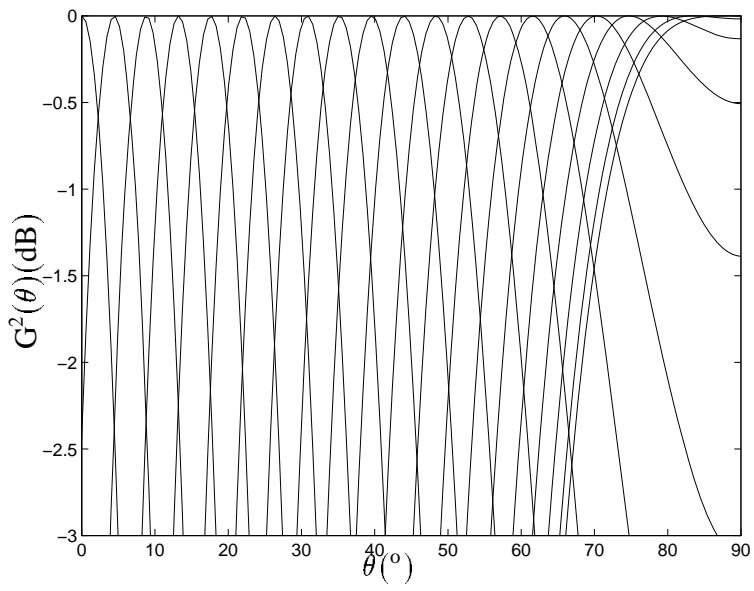

Figure 6. Beampointing patterns for all synchronous directions, $\mathrm{M}=\mathbf{2 0}$ and $\mathrm{N}=7$ 